\title{
12. What is visual-numeric literacy, and how does it work?
}

\author{
Elise Seip Tønnessen
}

\begin{abstract}
This article explores the concept of literacy related to the use of data visualizations. Literacy is here understood as the ability to make sense from semiotic resources in an educational context. Theoretically the discussion is based in social semiotic theory on multimodality in the tradition of New Literacy Studies. Empirical examples are taken from observations in two Social Science classrooms in upper secondary school in Norway, where the students work with publicly available data visualizations to answer tasks designed by their teacher. The discussion sums up factors that affect reading and learning from such complex resources: taking time to explore axis system, variables, and digitally available options; questioning data; and contextualizing results.
\end{abstract}

Keywords: Literacy; Numeracy; Multiliteracies; Reading for learning

\section{Introduction}

The development of innovative data visualizations creates new demands on the ability to make meaningful use of such resources. This ability may be seen as a kind of literacy, which requires certain skills that may be related to the meaning-making resources applied, to the digital technology, and to the understanding of specialized conventions in statistics. This article will explore this concept of literacy theoretically and discuss it in light of empirical examples. The examples were observed in Social Science classrooms in upper secondary schools in Norway. The students were asked to use digital data visualizations to answer specific questions and complete tasks designed by the teacher. The empirical cases will be used to discuss these literacy

Engebretsen, M. and H. Kennedy (eds.), Data Visualization in Society. Amsterdam: Amsterdam University Press, 2020 DOI 10.5117/9789463722902_CH12 
practices, and how they appear as strong, weaker, or even failing in relation to the planned learning outcomes. My aim is to explore the relationships between the understanding of semiotic modes, of how digital media work, and more specifically of how familiar the students are with conventions for statistics and visual graphs.

\section{Theoretical perspectives}

\section{Literacies}

The New London Group (1996) calls for a plural concept of multiliteracies to meet the challenges posed by new media and globalization in society. Gunther Kress (2003, p. 23), one of the participants in the New London Group, points to the complexity of literacies, claiming the concept needs to take into account the relevant semiotic modes as well as the ability to use media for production and distribution in multimodal communication. In this chapter I will explore literacy as cultural practices that are shaped by and adjusted to a certain context (Barton, 2007). My interest is in literacy practices, but empirically these can be studied through situated literacy events. The events studied in this chapter are situated in a school context, but the learning resources come from a research context, and are read in a digital medium. This complicated context is in line with the learning outcomes related to developing the students as 'budding researchers':

Students should be able to use a variety of digital search strategies to find and compare information that describes problems from different points of view and evaluate the objectives and relevance of one's sources. (KP, 2013, Social Science curriculum)

The use of the term literacy extended from verbal language to other semiotic systems and media has been criticized for its lack of precision (e.g. Kress, 2003, p. 23; Hasan, 1996). In this article I will take the idea of literacy as the ability to make sense from semiotic resources in an educational context (Hasan, 1996) as my starting point and discuss how this may be more accurately described based on my empirical examples. A preliminary label for this form of literacy may be visual-numeric literacy, which draws on an understanding that reading such graphs requires the mastery of certain modes, mainly visual and numeric. In previous research there is a tendency 
to focus either on the visual (Chevalier et al., 2018; Allen, 2018) or on the numeric dimension (Prince \& Archer, 2014) of related literacies.

The visual modes relevant to reading graphs are organized in a composition (van Leeuwen, 2005), where elements in a defined space make meaning in terms of size, direction, and relative distance to the axes defining the space. This connects to the numeric dimension, where specialized conventions have been developed within mathematics and statistics. Some of these are spatial conventions about how systems of axes or columns and rows work. They are connected to methodological conventions about relations between variables (independent and dependent) and how they are placed spatially, combined with more general conventions about what directions mean in our culture (such as developments in time moving from left to right, positive values moving upwards and to the right).

Such complexities can be comprehended on different levels. Ruqaiya Hasan (1996) distinguished between three aspects of literacy. Firstly, recognition literacy is necessary to understand the relevant meaning resources. For writing the central resource is the alphabet; in visual-numeric literacy relevant semiotic resources are e.g. lines, bars, bubbles, colours, and labels. In digital media it may also include knowing how to find the graphs and the options for changing them.

Hasan argues that recognition literacy, if taught in isolation, is not sufficient. Literacy also requires discursive abilities, connected to 'enabling the pupils to do something with their language' (1996, p. 399). This entails the ability to produce and interpret connected texts within the genre suited for the context in question. This is important to enable users to achieve their goals, whatever they may be, and be active participants in society. In visual-numeric literacy this may involve posing relevant questions and understanding how variables can be combined and how to choose displays that best visualize a point. Action literacy is developed through practice; reading several data visualizations gives the experience necessary to make meaningful choices.

The third aspect, reflection literacy, involves the ability to reflect, enquire, and analyse (p. 408). This includes reflection over reading practices in society, questioning the values they carry and the perspectives they entail. Reflection literacy is what it takes to question choices and readings, critique sources, and contextualize findings. These three aspects of literacy are interrelated. Hasan claims that 'reflection literacy includes a well-informed variety of action literacy $[. .$.$] and the latter includes recognition literacy; the reverse$ is, however, not true' (p. 417). 


\section{Case study}

The empirical examples for my discussion were observed in two classrooms in secondary schools in Norway. In both cases the subject was Social Studies, with a basic course for students aged 17 in case $A$ and an advanced course in Human Geography for students aged 18 in case B. In both cases we visited the classroom to observe the students working with tasks that were a normal part of teaching, planned by their teacher. In case A the class used Google Public Data (2018) in their work on unemployment, as part of the topic 'Working life and business'. The tasks were integrated in a lecture where the teacher introduced the topic before the students went online, and afterwards he summed up the findings in a classroom dialogue. In case B the topic was 'Demography', and the tools used were from Gapminder (2018). The class had spent one lesson getting acquainted with the tool previously and handed in their findings in writing after the double lesson spent on the tasks.

The data visualizations used in these two cases are both available to the general public. The unemployment graph in Google Public Data is based on big datasets from Eurostat, displaying data on unemployment rates as a line chart, showing time on the $\mathrm{x}$-axis and percentage of unemployed on the y-axis (Figure 12.1). The tool includes three other options for visualization: bar charts, bubble charts, or maps, but none of these were used in the classroom we observed.

The Gapminder tools used in case B are developed to visualize publicly available data in order to promote 'a fact-based worldview' (Gapminder, 2018) among the general public and include instructions for teachers. Links to Gapminder are included among the external resources suggested for Social Studies by the National Digital Learning Arena, the official portal for digital learning materials in Norwegian upper secondary schools (NDLA, 2018). The default settings display the relations between income ( $\mathrm{x}$-axis) and life expectancy (y-axis) as a bubble chart (Figure 12.2). In addition to the relations between the two axes, it offers coding of the bubbles in size and colour (the default settings of size indicating population size and colour indicating world region may be changed in the search fields to the right, Figure 12.2). In addition, a time dimension is shown as an animation. The tool includes options for various ways of displaying the data: trends (line charts), ranks (bar charts), maps, population pyramids, and stacked area graphs, but none of these were activated in the classroom we observed.

In each class students volunteered to take part in the study, 8 students in case A and 10 students in case B. The study followed the standards of Norwegian ethical guidelines and was approved by the Data Protection 


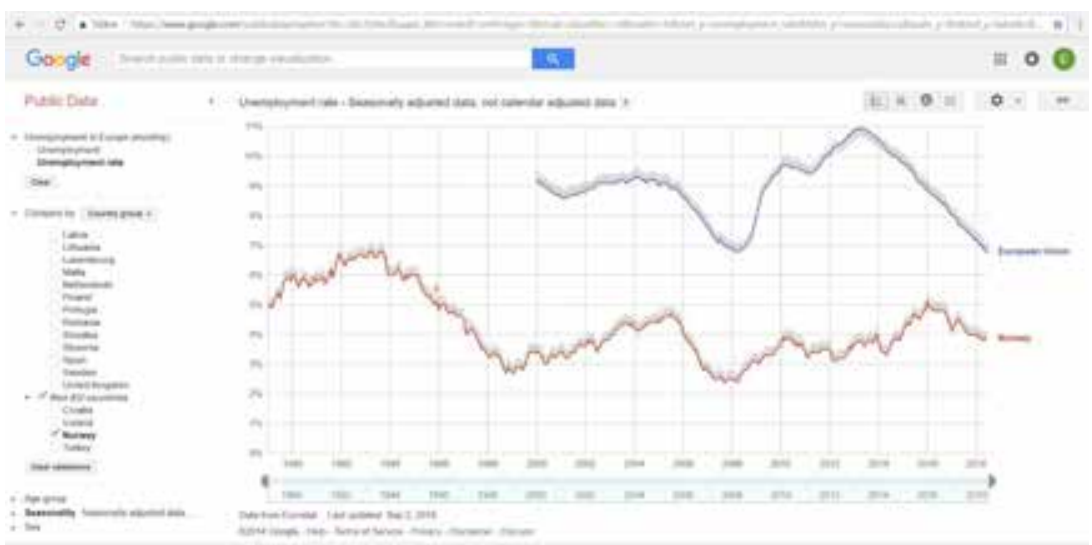

Figure 12.1. Screenshot of Google Public Data. Based on free material from Google Public Data. Source of data: Eurostat, CC-BY licence.

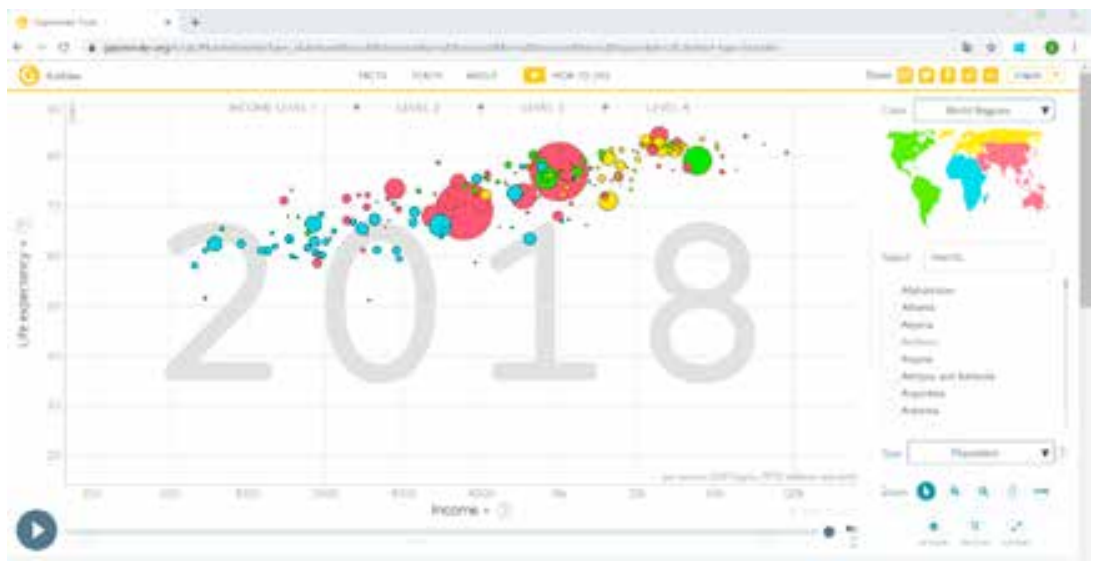

Figure 12.2. Screenshot of the starting image on Gapminder tools. Based on free material from gapminder.org, CC-BY license.

Authorities in Norway. The students worked in pairs with the tasks given by the teacher, and we made screen recordings and recordings of the pairs and their discussions while working.

In the analysis my focus is on how working with these data visualizations may contribute to learning, and on identifying factors that enhance learning, or represent obstacles to learning. My understanding of learning is inspired by Bezemer and Kress (2016) who emphasize that learning requires engagement and rests on interpretation:

Instead of measuring the transmission of knowledge, our interest is in uncovering and describing the transformative principles that learners 
bring to bear as they engage with the world around them. (Bezemer \& Kress, 2016, p. 38)

Hence, when learners engage with the world through textual and mediated means, learning is closely connected to literacy, to handling the semiotic resources, and in our cases the digital media involved. As a basis for the analysis below, I looked systematically through the screen recordings, noting which semiotic and digital resources were used, how they were interpreted, and how they contributed to completing the tasks given by the teacher. This allowed me to point out factors that lead to more or less meaningful literacy practices.

\section{Analysis of literacy practices}

Out of the nine pairs we observed in the two cases, most of them worked steadily through the lesson to answer the questions designed by the teacher. Analysing the literacy practices, I assessed them as situated in an educational setting, where success is seen in relation to learning, understood as active engagement in transformative processes in line with Bezemer \& Kress (2016). I found instances of successful readings as well as weaker readings or direct misreadings in each literacy event. In the following I will explore the factors leading to good, weak, or failed reading events across the groups.

\section{What characterizes successful literacy practices?}

The best practices I observed were characterized by the students taking the time to understand how the graphs worked before they started exploring them and answering the specific tasks designed by the teacher. In case B the two girls in group 2 started by asking what the colours of the bubbles in Gapminder stood for and agreed that it indicated on which continent the country was located. Group 5 used this knowledge to ascertain the location of countries with which they were not familiar. Group 3 took time to check that they understood the labels in the axis system, translating from English to Norwegian.

In case A I also found instances where the students posed questions relevant to reading the graphs. Group 7 asked the teacher how they could access data from before 2000, which was the starting point for EU data, and he helped them discover that some countries were represented with a longer time span. They also asked about the difference between 'unemployment' 
(total number) and 'unemployment rate' (percentage of work force unemployed), but in this case they were just told to choose the latter, not to investigate the help information available by clicking the question mark besides the label. These examples show that a basic factor in visual-numeric literacy is getting an overview of the composition of graph, variables, and options included.

In case B one of the tasks was to reflect upon why some of the bubbles in Gapminder were not moving during the first part of the time series from 1800 to 2015. Two of the groups passed quickly over this question by saying that it meant no change. But three groups questioned whether there were data available for all countries back in the 180os. Group 2 ran the relevant time series a couple of times to determine which countries this applied to, and found that it was mostly African countries, where public statistics may not go that far back. However, none of the groups consulted the label 'data doubts' (bottom, right-hand side, Figure 12.2). If they had, they would have found the information that 'countries on a lower income level have lower data quality in general, as less resources are available for compiling statistics. Historic estimates before 1950 are generally also more rough' (Gapminder, 2018). These examples show the need for critical assessment of the numbers and statistics behind the graphs, which may be supported by information not immediately visible on the screen. Hence it also points to the need to understand the relationship between what is available at the (screen) surface of digital texts and what may be accessed through links and clicking.

As can be seen from these examples, meaningful readings depend on background information. While exploring the graphs, the students leaned on their previous knowledge about society and history. In general, these were not very sophisticated, which is not surprising given their young age. The students in case B related what they saw to well-known historic events such as the World Wars, or the Wall Street Crash of 1929. The students exploring the unemployment rates in case A were aware of the financial crisis, and how it affected Greece in particular, but their background knowledge was more approximate when it came to what caused the crisis, and how this connected to unemployment rates.

How the tasks were designed carried consequences for how the readers engaged with the graphs, both in terms of personal engagement and background knowledge. In case A the first question was about comparing the unemployment rate now to when the teacher was young in the 1990s. When group 9 compared the 6.7 percent unemployment in 1994 to the recent rise from 3.2 percent to 4.9 percent $(2014-2016)$ they reflected: 'We 
think there is a crisis now, but it was so much higher then!' In this case the personal contextualization provided a longer time span for assessing the numeric information.

Whereas the students in general used the data visualizations mainly to confirm and — at best—expand the knowledge they already possessed, one example illustrates how the teacher designed a task that encouraged the students to learn something new from data visualizations. They were asked to focus on China in the time span from 1957 to 1962 and were specifically challenged to search for information about the great famine following from Mao's agriculture and industry reform policy. For most of the groups this led to reasoning about how natural conditions in combination with politics may affect ordinary people. For group 5 this led to emotional responses as they realized the suffering involved. Going back to the graph after updating their background knowledge, they followed the big pink bubble as it bounced downwards to indicate the fall in life expectancy, along with a left move to indicate a parallel fall in income. They were touched by the facts:

- Wow, that was a lot [1958]

- Yes. [moving forward to 196o]

- Oops!

- Yeah, there was a famine!

- But a life expectancy of 30 years. How is that possible?

- It is quite sick!

Concluding from these examples, I find literacy practices that enable the students to expand and reflect on their knowledge when they establish an understanding of how the variables and values on the axes define the graphic space. Furthermore, these readings were characterized by an active engagement in the topics studied, where the students formulated their expectations in advance, based on prior knowledge, while they were still open to include new information and reflect about reasons and consequences. A distinctive feature was that the students posed questions and spent the time and effort it took to find the answers, combining the displayed data with supplementary information.

\section{Why do some readings appear weaker?}

The most prominent trend in our observations seemed to be literacy practices that did not harvest the full learning potential from the data visualizations. This has to do with the specific skills required in visual-numeric literacy, but also with the readers' degree of engagement with the graphs and the 


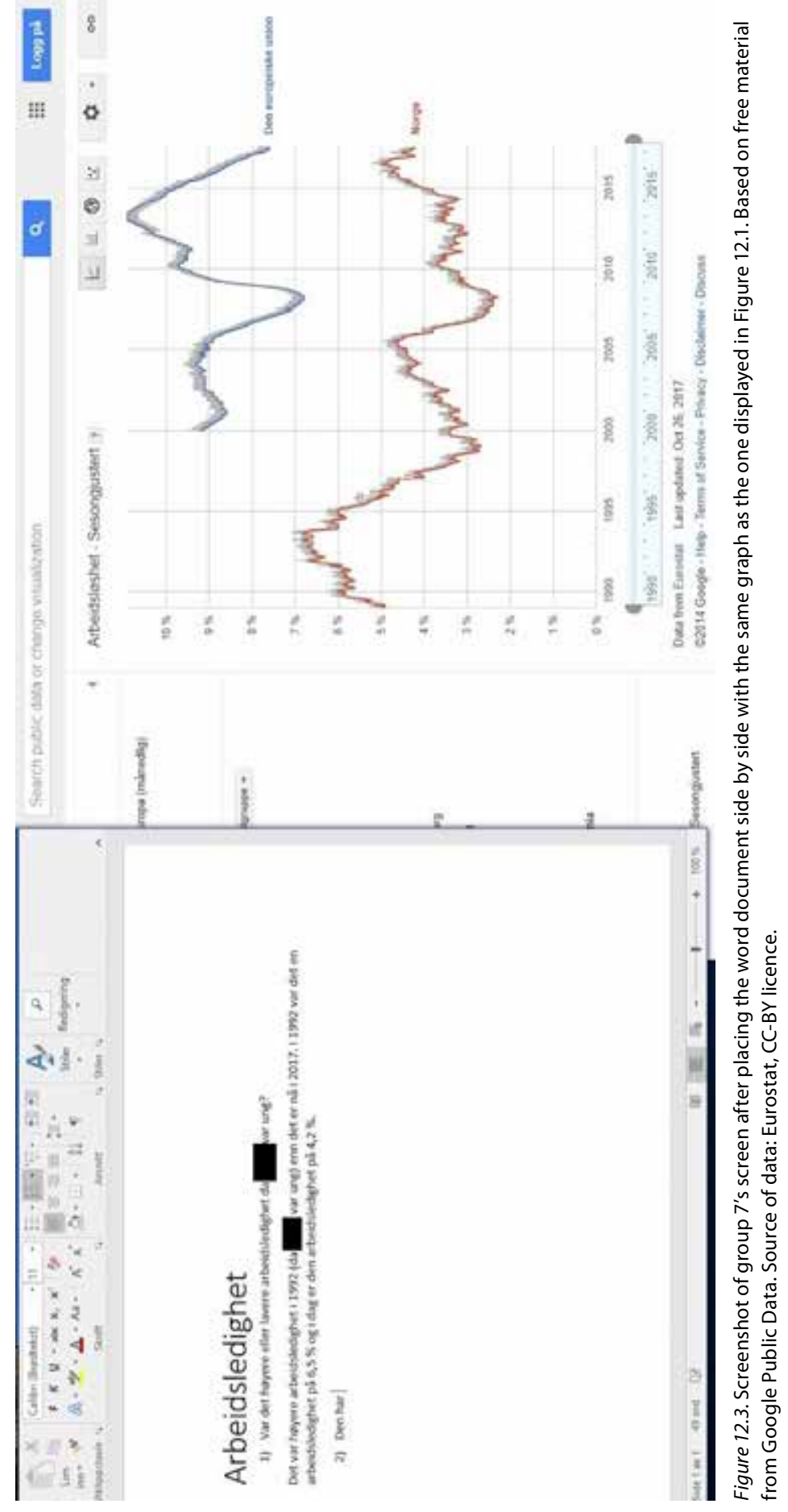


tasks given by the teachers. When the students in case A first opened the graph on unemployment, they were typically looking for sudden turns and dramatic changes. Their engagement increased when they detected crises in Greece, Spain, Iceland, or Estonia around 2008. However, they seemed to be more interested in the changes as such than in the level of unemployment over time. Group 7 at first estimated the unemployment rate in Norway to be quite stable. Then they decided to place the Word document where they were typing the answers side by side with the graph on the screen. This led to a compressed $\mathrm{x}$-axis that made the slope of the rising and falling curves steeper (Figure 12.3). Not taking this relative change into consideration, the girl who was typing exclaimed: 'Why did I say it was stable?' and they adjusted their answers accordingly. If they had compared the variation observed with unemployment rates from earlier years, or in other countries, they might have modified their assessment, as another group did when asserting that the unemployment rate in Norway was overall lower than in other countries or regions.

The tendency to extract the most visible facts from the graph without seeing them in relation to other available information was even more striking for the groups working on Gapminder, since this tool contains more information and more options for display. When the students first approached the bubble chart with the default settings of income (x-axis) and life expectancy (y-axis), they focused mainly on the extreme cases; the lowest or highest life expectancy or income, and when they moved on to the following tasks, the highest child mortality and fertility rate. This led to readings that picked out single facts, rather than discovering trends.

Several examples of such isolated readings were observed. In case A I found that the students described the development in countries one by one, apparently not noticing the option to compare groups of countries (upper left corner in Figure 12.1). When the students in case B were asked to comment on the connections between income and life expectancy, most of them just asserted that the better the income the longer the life expectancy. Only a few formulated reasons, e.g. how better economy allows for better healthcare. They were also asked to find the four countries with lowest life expectancy today and reflect on which parts of the world they could be found in. Answering that three of them (Lesotho, Swaziland, and Central African Republic) were in Africa and one (Afghanistan) was in Asia did not really do credit to the level of detail included in the tool they were using. And when they were asked to compare the development of child mortality for three countries (USA, Norway, and Mali), they mostly described the countries one by one, rarely commenting on the relations between them. 
Furthermore, I found few examples where the students reflect on the meaning of the values on the axes. Even though the teacher in case B specifically told them to note that the values for income on the $\mathrm{x}$-axis were logarithmic (each interval doubles the value), they did not question what this meant and how it affected the shape of the graph. When they changed the axes to child mortality and time, they did not notice that now the y-axis had a logarithmic scale. When discussing child mortality, they did not seem to take in the realities of the measurement: ' $0-5$ year olds dying per 1,00o born' (explanation along the y-axis). In the case of Mali this meant that every second child died before the age of 5 throughout the nineteenth century, and the situation did not improve until well into the 1960s (Figure 12.4a).

One reason why the students rarely exhausted the full potential of the graph may be that they did not take the time to get fully acquainted with it. Several functions were never activated, such as the background information marked with a question mark where there are options for choice, or the information videos placed right underneath the graph.

I did, however, find a few examples of students discussing the meaning of the labels. This occurred when the wording on their task sheet was not exactly the same as on the screen. The students in group 5 discussed whether there was a difference between 'Children per woman', which was the label used in task 3, and 'Babies per woman', which was the label they found in Gapminder. One of the boys claimed that the term babies was limited to the first year in life, while children would be used for those past age one. This was knowledge from the textbook, and it would have been relevant for the variable 'child mortality', which was used in task 2.

The main factor weakening these reading events was the lack of time and effort invested in reading and interpreting the visualizations and the data they were based on. These data visualizations are packed with information and require careful and thorough interpretation. The combination of several variables in one display is its specific strength, but this strength was not exploited to its full extent in the cases I observed. From my observations it seems relevant to ask how many dimensions the students are able to take in at once. In the cases I observed, none of the students finished all the tasks given by the teacher. This meant that they never got to the stage where they were allowed to pose their own questions to the data, which is the learning outcome envisioned in the national curriculum. Hence the time available compared to the workload would seem to suggest that quick reading and short answers are more realistic outcomes. 


\section{A specific case of misreadings}

There were not many direct misunderstandings in our examples. But one specifıc task in case B led to a row of very different choices that it is illuminating to study in depth. The misreadings happened when the students were asked to change the variables on the axes. The task formulated by the teacher said:

2. Choose the indicator Time on the first axis and Child Mortality on the second axis.

a) Describe how child mortality has developed in the USA, Norway, and Mali.

The problem appeared when the students had difficulties finding the small triangle next to the labels that allowed them to choose other variables. The resulting graphs can be seen in Figure 12.4a-d. Group 2 and 3 established the graph with the intended axes variables on their first try (Figure 12.4a) and had no specific difficulties reading the graph. Displaying time on the $\mathrm{x}$-axis made it easy to see development over time. They commented on the general trend that child mortality had been lower and decreased more rapidly in Norway and the USA than in Mali, and questioned why the curve for Norway had so many ups and downs throughout the 180os. Group 2 also questioned the sudden rise in child mortality in the US in 1918 and found the explanation through a search that led them to information about the Spanish flu.

Group 1 searched for 'life expectancy' in the search field for coding the size of the bubbles (bottom, right), and ended up changing this to 'number of child deaths', and not changing the axes variables (Figure 12.4b). In the resulting graph child mortality was indicated in total numbers by the size of the bubbles. At the time the screenshot was taken, they approached the teacher to ask why Mali was not moving at all. She directed them to the right axis variables, resulting in Figure 12.4a. The next time they needed to change the axes for task 3 , they had no trouble applying this literacy skill to a new task.

Group 4 got the axes mixed up; they changed the y-axis to 'Time' and the $\mathrm{x}$-axis to 'Child mortality'. One of them suggested that it would be more natural to have Child mortality on the vertical axis, but after some changes back and forth they ended up with the graph in Figure 12.4c. Displaying time on the y-axis is counter-intuitive to established conventions of reading time development from left to right (Kress \& van Leeuwen, 2006). In addition, the value on the $\mathrm{x}$-axis was negative, which meant that the movement over time in this graph went from bottom right to top left. Within Western reading 

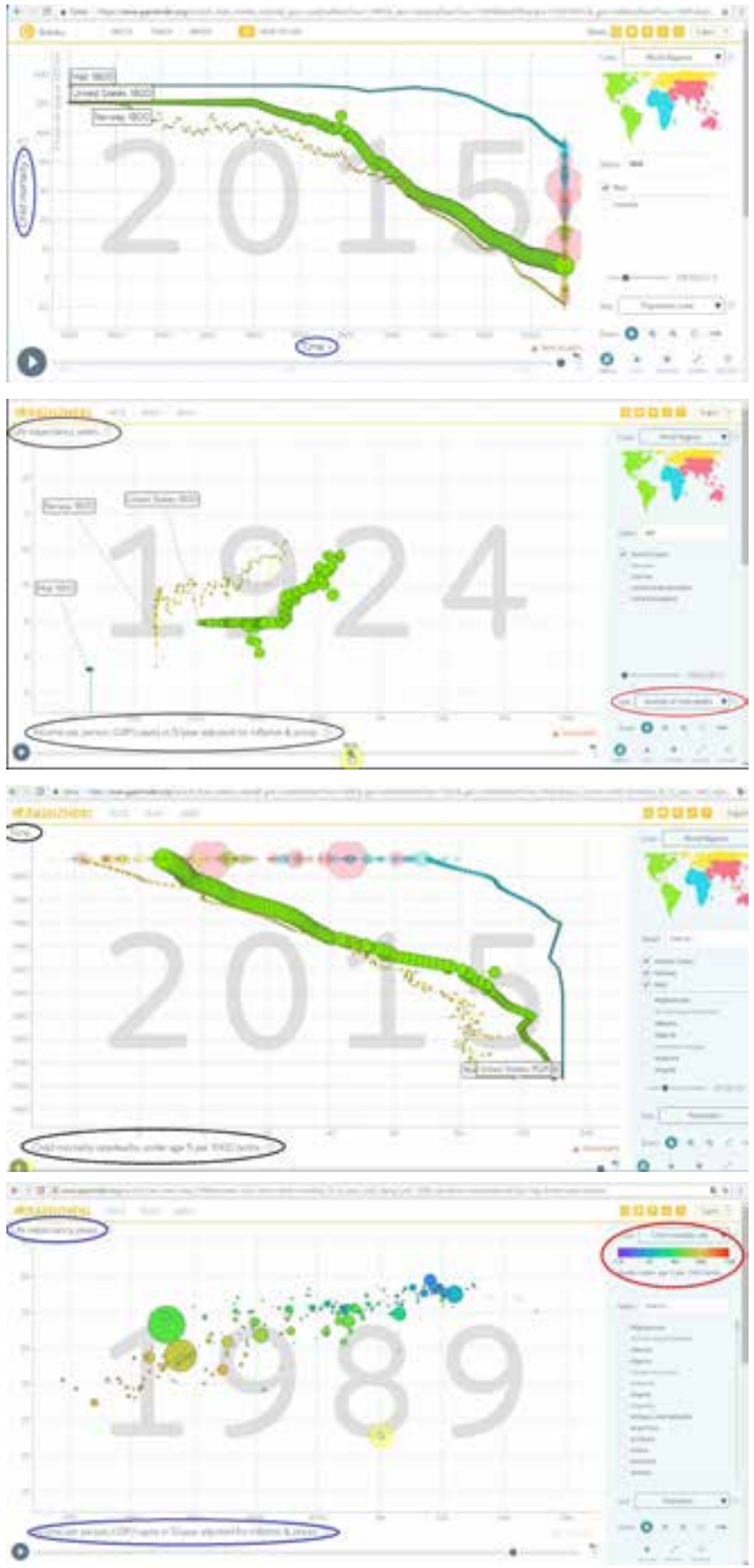

Figure 12.4 Versions of graph to answer task 2 on child mortality in three countries. a) Group 2 and 3 with intended axis variables, b) Group 1, c) Group 4, d) Group 5. Based on free material from gapminder.org, CC-BY licence. 
conventions this is hard to interpret. Due to this confusion, and time limits, the group ended up not answering question 2 .

Group 5 also had problems changing the axes variables, and although one of the students questioned the result, they did not proceed to finding out what the problem was. They searched for child mortality in the search field for colour-coding of the bubbles, resulting in Figure 12.4d. Here child mortality was visualized in colour, indicating high mortality with warm colours and low mortality with cold colours. Keeping income and life expectancy as axes values resulted in a rising pattern of bubbles. In their discussion the boys talked about Norway and the USA 'peaking upwards', and in writing they first formulated the rise as an improvement: 'In the USA and Norway child mortality has developed steadily upwards', but then they corrected the last two words to 'in a positive direction'. Hence their answer appeared correct, but it was taken from their general knowledge rather than from their reading of the graph. The teacher would probably never know that they needed some instruction on the rather simple task of finding out how to change axis variables in this specific tool.

These misreadings are interesting since the problem is media-related rather than semiotic in nature. The options for choosing variables and coding are inherent in the dynamics of digital media that afford exploratory work with data visualizations. The problems in our case B would not occur in a textbook where the display of graphs is stable and designed by experts for explanatory use. The more options given to the reader, the more demanding it gets to establish a graph that can be meaningfully read. In the classroom, misreadings are mostly avoided because the students are led by hand through the tasks designed by the teacher, but the independent and actively researching student envisioned in the national curriculum needs to understand which variables can be meaningfully combined and what forms of display will give a clear visualization. More experience with data visualizations is needed to foresee the results of chosen values, and consequently to be able to discover and correct mistakes. The ability to notice mistakes, and to analyse and correct them, and generally question readings, is vital to any kind of literacy (Roe, 2008, p. 96).

\section{Concluding discussion}

Our observations include groups on different levels, one working in a basic course, the other on a more advanced level. The students in case B demonstrate a higher level of literacy in their ability to activate their 
pre-understanding and contextualize their reading of the graphs. Still, this does not prevent them from encountering problems when they are asked to change the axis system and explore new datasets. One might argue that what I have termed 'misreadings' in this article is mainly due to students' problems in handling the many choices given by the digital Gapminder tool. This finding means that the literacy discussed in this article is not merely visual and numeric, it is also about how digital media work, and how they allow the user to interact with preprogrammed affordances in data visualizations. Consequently, the literacy I gave the preliminary label 'visual-numeric' may be more complex than this term suggests.

This complexity involves connections of statistic, technological, and semiotic resources that work on different levels. On a fundamental level, the axis system defines a space that is semiotically charged, and hence functions as an overall framework for reading the graph. Within this framework the lines and bubbles require the reader to take notice of slope and area respectively, and also codings of colour (Cairo, 2016, p. 128). Interpreting or producing a meaningful space between the axes requires specialized statistical knowledge of variables, values, and other conventions. The digital medium is the means to systematize, save, and reshape data, often too big to handle in any other medium, but also to display and interact with these data. This requires both general and more specialized digital literacy.

As pointed out by Hasan (1996), literacy works on different levels. The students recognized several semiotic resources and digital functions from their general experience with digital media, e.g. using search functions, pressing the play button. They may have recognized the triangle opening the menu of variables (see arrow in Figure 12.2) if it had been shown to them when needed. But this simple act of recognition is related to a more general insight in how digital media facilitate access to layers of information behind the screen surface.

The action aspects of literacy seem to need guidance and teaching in our example. The teachers designed a progression of tasks to build experience for the tasks to come, e.g. in case B asking why some bubbles were not moving in the early years, before the students approached the task of comparing Mali to two Western countries. Our observations reveal a need to find teachable moments in school literacy practices. One appeared when the students first were asked to change the axis variables. Those students who had the teacher's attention at that moment avoided 'misreadings' and carried this understanding on to the following tasks.

Reflection literacy involves the ability to critically question the ways data are presented, how they are used, and what for, and also to question one's own 
reading practices. In the misreadings I observed, some of the students did pose questions, but they rarely went back to correct their mistakes. Maybe this was because of time limits, or maybe the framing of tasks in the school context directed the attention to get the tasks done, more than to in-depth reading. In the cases I studied, the learning objectives were directed towards subject knowledge in Social Studies, rather than to developing the students' specialized literacy for reading digital graphs. As pointed out in my introduction, the curriculum encourages a focus on literacy integrated in other learning outcomes. Amid everyday classroom demands this double focus seems hard to maintain. This points to a need for special attention towards literacy even in secondary schooling, including basic skills in using visual, numeric, and verbal resources as well as digital media (Norwegian Directorate for Education and Training, 2013).

My discussion of best, weaker, and failing practices should not be taken as authoritative universals; each literacy event must be understood in context. In a different situation the objectives of reading or the data visualizations read may justify a more critical, or even subversive, literacy practice. Some of the experiences from my classroom observations may still be transferable, such as the time it takes to get acquainted with the graph and the digital options it affords; the need to question underlying data; and the challenge of contextualizing what is being displayed. Considering the increased use of data visualization in society, the curriculum's ambitions to teach students search strategies, in combination with the ability to evaluate the objectives and relevance of one's sources, seems vital to lifelong learning.

\section{Acknowledgements}

This research has been funded by Research Council of Norway through the INDVIL project. A special thanks to my colleague, Professor Anne Løvland, for taking part in developing the research design and assisting in collecting the data.

\section{References}

Allen, W. L. (2018). Visual brokerage: Communicating data and research through visualisation. Public Understanding of Science. Advance online publication. https://doi.org/10.1177/0963662518756853

Barton, D. (2007). Literacy: An introduction to the ecology of written language. Malden: Blackwell Publishing. 
Bezemer, J., \& Kress, G. (2016). Multimodality, learning and communication: A social semiotic Frame. London \& New York: Routledge.

Cairo, A. (2016). The truthful art:Data, charts, and maps for communication. Berkeley, CA: New Riders.

Chevalier, F., Henry Riche, N., Alper, B., Plaisant, C., Boy, J., \& Elmqvist, N. (2018). Observations and reflections on visualization literacy in elementary school. IEEE Computer Graphics and Applications, 38(3), 21-29. http://doi.org/10.1109/ MCG.2018.032421650

Hasan, R. (1996). Literacy, everyday talk and society. In: R. Hasan \& G. Williams (Eds.), Literacy in society. (pp. 377-424). London: Longman.

Gapminder (2018). Downloaded April 26, 2018 from https://www.gapminder.org/ tools/\#_data_/lastModified:1524739828963\&lastModified:1524739828963;\& chart-type=bubbles

Google Public Data (2018). Unemployment rate in Europe [Data Set]. Downloaded April 26, 2018 from https://www.google.com/publicdata/ explore?ds=z8o7pt6rd5uqa6

Kress, G. (2003). Literacy in the new media age. London \& New York: Routledge.

Kress, G., \& van Leeuwen, T. (2006). Reading images: The grammar of visual design (2nd ed.). London: Routledge.

Norwegian Directorate for Education and Training (2013). Social Studies Subject Curriculum (SAF1-03 / Samfunnsfag). The Knowledge Promotion Reform. Revised 2013. Retrieved April 23, 2018 from https://www.udir.no/klo6/SAF1-03

NDLA (2018). Gapminder. Retrieved August 1, 2018 from https://ndla.no/nb/ node $/ 7660$ ?fag $=36$

Prince, R., \& Archer, A. (2014). Exploring academic voice in multimodal quantitative texts. Literacy and Numeracy Studies, 22(1), pp. 39-57. https://doi.org/10.5130/ lns.v22i1.4178

Roe, A. (2008). Lesedidaktikk-etter den første leseopplceringen. [Reading didactics-After the initial teaching of reading]. Oslo: Universitetsforlaget.

van Leeuwen, T. (2005). Introducing social semiotics. London \& New York: Routledge

\section{About the author}

Elise Seip Tønnessen is Professor in the Department of Nordic and Media Studies, University of Agder, Norway. Her research interests range from literacy across modes and media, to reception studies in educational as well as leisure time settings. She has published on literacy, children's media culture, and children's literature. 
